\title{
Article \\ Echocardiographic Assessment of Right Ventricular-Arterial Coupling in Predicting Prognosis of Pulmonary Arterial Hypertension Patients
}

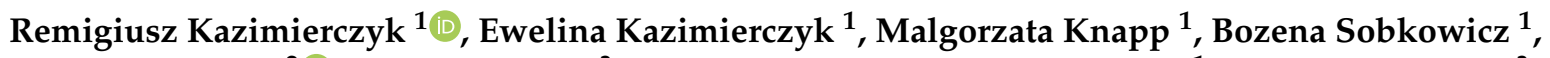 \\ Lukasz A. Malek ${ }^{2}$ D, Piotr Blaszczak ${ }^{3}$, Katarzyna Ptaszynska-Kopczynska ${ }^{1}$, Ryszard Grzywna ${ }^{3}$ \\ and Karol A. Kaminski 1,4,*(D)
}

check for updates

Citation: Kazimierczyk, R.; Kazimierczyk, E.; Knapp, M.; Sobkowicz, B.; Malek, L.A.; Blaszczak, P.; Ptaszynska-Kopczynska, K.; Grzywna, R.; Kaminski, K.A. Echocardiographic Assessment of Right Ventricular-Arterial Coupling in Predicting Prognosis of Pulmonary Arterial Hypertension Patients. J. Clin. Med. 2021, 10, 2995. https:// doi.org/10.3390/jcm10132995

Academic Editor: Francesco Giallauria

Received: 22 June 2021

Accepted: 30 June 2021

Published: 5 July 2021

Publisher's Note: MDPI stays neutral with regard to jurisdictional claims in published maps and institutional affiliations.

Copyright: (c) 2021 by the authors. Licensee MDPI, Basel, Switzerland. This article is an open access article distributed under the terms and conditions of the Creative Commons Attribution (CC BY) license (https:// creativecommons.org/licenses/by/ $4.0 /)$.
1 Department of Cardiology, Medical University of Bialystok, 15-089 Bialystok, Poland; remigiuszk1989@gmail.com (R.K.); eidzkowska@wp.pl (E.K.); malgo33@interia.pl (M.K.); sobkowic@wp.pl (B.S.); kasia.ptaszynska@op.pl (K.P.-K.)

2 Department of Epidemiology, Cardiovascular Disease Prevention and Health Promotion, National Institute of Cardiology, 04-628 Warsaw, Poland; lukasz.a.malek@gmail.com

3 Department of Cardiology, Cardinal Wyszynski' Hospital, 20-718 Lublin, Poland; blaszcz12345@interia.pl (P.B.); grzywna@gmail.com (R.G.)

4 Department of Population Medicine and Lifestyle Diseases Prevention, Medical University of Bialystok, 15-089 Bialystok, Poland

* Correspondence: fizklin@wp.pl

\begin{abstract}
In response to an increased afterload in pulmonary arterial hypertension (PAH), the right ventricle (RV) adapts by remodeling and increasing contractility. The idea of coupling refers to maintaining a relatively constant relationship between ventricular contractility and afterload. Twenty-eight stable PAH patients (mean age $49.5 \pm 15.5$ years) were enrolled into the study. The follow-up time of this study was 58 months, and the combined endpoint (CEP) was defined as death or clinical deterioration. We used echo TAPSE as a surrogate of RV contractility and estimated systolic pulmonary artery pressure (sPAP) reflecting RV afterload. Ventricular-arterial coupling was evaluated by the ratio between these two parameters (TAPSE/sPAP). In the PAH group, the mean pulmonary artery pressure (mPAP) was $47.29 \pm 15.3 \mathrm{mmHg}$. The mean echo-estimated TAPSE/sPAP was $0.34 \pm 0.19 \mathrm{~mm} / \mathrm{mmHg}$ and was comparable in value and prognostic usefulness to the parameter derived from magnetic resonance and catheterization (ROC analysis). Patients who had CEP $(n=21)$ had a significantly higher $\mathrm{mPAP}(53.11 \pm 17.11 \mathrm{mmHg}$ vs. $34.86 \pm 8.49 \mathrm{mmHg}, p=0.03)$ and lower TAPSE/sPAP $(0.30 \pm 0.21$ vs. $0.43 \pm 0.23, p=0.04)$. Patients with a TAPSE/sPAP lower than $0.25 \mathrm{~mm} / \mathrm{mmHg}$ had worse prognosis, with log-rank test $p=0.001$. the echocardiographic estimation of TAPSE/sPAP offers an easy, reliable, non-invasive prognostic parameter for the comprehensive assessment of hemodynamic adaptation in $\mathrm{PAH}$ patients.
\end{abstract}

Keywords: echocardiography; coupling; pulmonary arterial hypertension; prognosis; right ventricle

\section{Introduction}

Pulmonary arterial hypertension $(\mathrm{PAH})$ is a rare disease that causes increased pulmonary vascular resistance (PVR) and elevated pulmonary arterial pressures (PAPs). This often leads to heart failure and premature death [1]. Due to the progressive course of the disease, maladaptive right ventricular (RV) dilatation and/or hypertrophy occurs, eventually resulting in RV failure [2,3]. The development of RV failure carries a two- to threefold increase in risk of cardiac death, irrespective of the degree of left ventricular (LV) dysfunction $[4,5]$.

When the RV becomes overloaded in PAH, it mostly adapts to the increased afterload by increasing contractility according to Frank-Starling law. However, with the depletion of systolic function, the RV becomes uncoupled from pulmonary circulation and dilates to 
preserve flow output [2]. The best method (gold standard) to assess cardiac contractility is pressure-volume loop-derived end-systolic elastance (Ees). RV-arterial coupling is then calculated as the ratio of end-systolic elastance to arterial elastance (Ees/Ea) [2,3]. However, obtaining Ees and Ea with the use of pressure-volume loops is time consuming and requires an invasive procedure. There is still a need for accurate, noninvasive measurements of RV function, which might provide prognostic information useful for finding the group of patients needing therapy escalation, even before clinical deterioration. Previous studies confirm several noninvasive parameters of RV function as important prognostic factors in PAH, e.g., echocardiographic tricuspid annular plane systolic excursion (TAPSE); pulmonary arterial systolic pressure (sPAP) [4-6]; MRI-derived right ventricle ejection fraction; and standardized glucose uptake of RV in PET analysis [7]. The ratio of TAPSE/sPAP, introduced earlier, has been proposed as an index of in vivo RV shortening in the longitudinal axis versus developed force in patients with chronic heart failure, and was found to be a non-invasive, indirect measurement of RV contractile function and RV-arterial coupling $[6,8,9]$. It seems that this approach to the estimation of RV-arterial coupling may have functional and prognostic relevance, especially at bedside.

In this study, we aimed to (1) verify the relationship between the echo-derived RVarterial coupling surrogate, TAPSE/sPAP ratio, and hemodynamic parameters obtained from right heart catheterization (RHC) and magnetic resonance imaging (MRI) in PAH patients, and (2) investigate whether the echocardiographic TAPSE/sPAP ratio has prognostic significance in $\mathrm{PAH}$ patients.

This paper presents an echo-focused analysis (with 58 months of follow-up observation) of a PAH group enrolled to the project, with a grant entitled "The role of PET/MRI hybrid imaging in assessment of pulmonary arterial hypertension patients", of which partial results were previously published $[7,10,11]$.

\section{Materials and Methods}

\subsection{Population Characteristics}

Into the study we enrolled twenty-eight clinically stable PAH patients. Pre-capillary pulmonary hypertension was confirmed by RHC (mean pulmonary artery pressure (mPAP) $\geq 25 \mathrm{mmHg}$, pulmonary artery wedge pressure (PAWP) $\leq 15 \mathrm{mmHg}$ ). According to European guidelines [1], we ruled out secondary $\mathrm{PH}$ causes. The exclusion criteria were the following: age below 18 years old, IV WHO class, PAH associated with prevalent systemic-to-pulmonary shunts due to moderate to large defects (according to European guidelines) [1], group II, III, IV, V of pulmonary hypertension, Eisenmenger physiology and contraindications to cardiac MRI. The control group consisted of twelve healthy controls who were matched based on sex and age. During the baseline evaluation, we performed a physical examination, six-minute walk test, cardiopulmonary exercise test (study group) and laboratory tests, e.g., serum B-type natriuretic peptide (BNP).

The clinical follow-up lasted 58 months. Death, WHO class worsening, and hospitalization due to pulmonary hypertension or right heart failure (requiring therapy with intravenous diuretics) were used as a combined clinical endpoint (CEP) for Kaplan-Meier analysis (as described before $[7,10,11]$ ).

The study was approved by the local Bioethics Committee. All patients gave written, informed consent for participating in the study, including the taking and storage of blood samples. The study complied with the Declaration of Helsinki.

\subsection{Echocardiography}

Echocardiographic examinations were performed in both study and control groups. Tricuspid annular plane systolic excursion (TAPSE) was obtained by M-mode imaging in the apical four-chamber view centered on the right ventricle. It was defined as the difference between the displacement of the RV lateral annulus from end-diastole to end-systole. The echo estimation of the sPAP (RVSP/esPAP) (reflecting RV afterload) was based on the sum of the peak velocity of tricuspid regurgitation (Bernoulli equation, TRPG $=4[\text { TRmax }]^{2}$ ) 
and of the estimated central venous pressure obtained by inferior cava vein diameter and collapsibility [12]. The echocardiographic mPAP was approximated from the esPAP using Chemla's formula: emPAP $=0.61 \times$ esPAP $+2 \mathrm{mmHg}$, as this formula had a good correlation and acceptable limits of agreement, as shown in [13-15].

\subsection{RHC/MRI Hemodynamic Parameters}

Right-sided heart catheterization (RHC) was performed using a Swan-Ganz catheter with standard technique [7], and during the procedure, several hemodynamic parameters were measured or calculated: pulmonary artery wedge pressure (PAWP); systolic, diastolic, and mean pulmonary artery pressure; cardiac output (calculated by thermodilution method); cardiac index; SV (obtained by dividing cardiac output by heart rate); right atrial pressure; and PVR, calculated as: (mean pulmonary artery pressure-PAWP)/cardiac output.

Cardiac magnetic resonance imaging is the gold standard noninvasive method for RV functional assessment. We used MRI scans to obtain TAPSE as a reference method to echo TAPSE [5]. MRI imaging was performed with a 3T Biograph mMR hybrid system (Siemens, Healthcare Erlangen, Erlangen, Germany). Systolic function assessment was based on SSFP short axis images from the tricuspid valve insertion point to the apex to encompass the entire RV. MRI and RHC were performed within a median of 4 days (2-6 days) in the PAH group at baseline visit.

\subsection{Statistical Analysis}

The data are expressed as a mean \pm standard deviation (SD) or median (interquartile range) in the case of variables with non-normal distribution (Kolmogorov-Smirnov test). The Student's $t$-test was used for continuous data and a $\chi^{2}$ test was used for categorical variables. Spearman's correlation coefficient was used to examine the relationship between two continuous variables. Receiver operator characteristic curves (ROCs) were plotted to determine the area under the curve (AUC) and the sensitivity and specificity of the optimal cut-offs. To investigate the occurrence of clinical endpoints, the Kaplan-Meier method with the log-rank test was implemented; $p<0.05$ was deemed statistically significant. A statistical software package, STATA13 (USA), was used for the analysis.

\section{Results}

\subsection{General Results}

The study group consisted of twenty-eight PAH patients, with the mean age $49.5 \pm 15.5$ years (idiopathic/heritable $n=19$; connective tissue diseases $n=4$; associated with congenital small/coincidental defects $n=5$ ). The control group consisted of twelve healthy volunteers, matched for age and sex (44.75 \pm 13.51 years, eight females). Five patients $(17 \%)$ were incident cases, while the rest of the study group were prevalent and receiving PAH-specific treatment at the time of study. Most of them were in the WHO functional class III $(64 \%, n=18)$ and the majority of patients were women $(60 \%, n=17)$. General groups characteristics are presented in Table 1 (the study group was also partially presented in our previous study [10]). 
Table 1. General characteristics of pulmonary arterial hypertension (PAH) patients and control group.

\begin{tabular}{|c|c|c|}
\hline & PAH Group & Control Group \\
\hline Patients, $n$ & 28 & 12 \\
\hline Age (years) & $49.5 \pm 15.5$ & $44.75 \pm 13.51$ \\
\hline Female sex, \% $(n)$ & $60(17)$ & $67(8)$ \\
\hline $\mathrm{BMI}\left(\mathrm{kg} / \mathrm{m}^{2}\right)$ & $24.5 \pm 3.7$ & $25.2 \pm 3.6$ \\
\hline 6MWD (m) & $392(322-453)$ * & $525(497-550)$ \\
\hline $\mathrm{BNP}(\mathrm{pg} / \mathrm{mL})$ & $310.8(54-3654) *$ & $27.2(13-42)$ \\
\hline \multicolumn{3}{|l|}{ Aetiology } \\
\hline IPAH/HPAH, \% (n) & $67(19)$ & \\
\hline CTDPH, \% (n) & $14(4)$ & \\
\hline CHDPH, \% (n) & $19(5)$ & \\
\hline \multicolumn{3}{|l|}{ Therapy } \\
\hline PDE5 inhibitors, \% $(n)$ & $38(10)$ & \\
\hline Prostacyclins, \% (n) & $20(5)$ & \\
\hline ERA, $\%(n)$ & $11(3)$ & \\
\hline Dual PDE5 inhibitor + ERA, \% $(n)$ & $31(8)$ & \\
\hline \multicolumn{3}{|l|}{ Echo parameters } \\
\hline RV basal diameter $(\mathrm{cm})$ & $6.6^{*}$ & $3.6 \pm 0.6$ \\
\hline $\operatorname{RAA}\left(\mathrm{cm}^{2}\right)$ & $25(19-32) *$ & $10(9-12)$ \\
\hline TAPSE (mm) & $19.84 \pm 4.28$ * & $26 \pm 3.4$ \\
\hline emPAP $(\mathrm{mmHg})$ & $47.29 \pm 15.3 *$ & $12.2 \pm 3.8$ \\
\hline $\operatorname{AcT}(\mathrm{ms})$ & $82 \pm 22 *$ & $130 \pm 28$ \\
\hline TAPSE/sPAP (echo) & $0.34 \pm 0.19$ * & $1.51 \pm 0.22$ \\
\hline \multicolumn{3}{|l|}{ RHC parameters } \\
\hline $\mathrm{sPAP}, \mathrm{mmHg}$ & $79.6 \pm 30.72$ & \\
\hline $\mathrm{dPAP}, \mathrm{mmHg}$ & $31.9 \pm 13.54$ & \\
\hline $\mathrm{mPAP}, \mathrm{mmHg}$ & $48.95 \pm 18.77$ & \\
\hline PAWP, mmHg & $10.5 \pm 2.03$ & \\
\hline $\mathrm{DPG}, \mathrm{mmHg}$ & $21.85 \pm 13.08$ & \\
\hline $\mathrm{CI}, \mathrm{L} / \mathrm{min} / \mathrm{m}^{2}$ & $2.65 \pm 0.60$ & \\
\hline $\mathrm{RAP}, \mathrm{mmHg}$ & $8.2 \pm 2.92$ & \\
\hline PVR, Wood Units & $9.17 \pm 5.69$ & \\
\hline \multicolumn{3}{|l|}{ MRI parameters } \\
\hline RVEF, \% & $44.91 \pm 7.9 *$ & $63.8 \pm 5.8$ \\
\hline $\mathrm{RV}$ EDV $/ \mathrm{BSA}, \mathrm{mL} / \mathrm{m}^{2}$ & $118.2 \pm 21.7 *$ & $73.6 \pm 12.2$ \\
\hline $\mathrm{RV} \mathrm{ESV} / \mathrm{BSA}, \mathrm{mL} / \mathrm{m}^{2}$ & $65.9 \pm 20.3^{*}$ & $28.2 \pm 9.6$ \\
\hline $\mathrm{RV}$ mass/BSA, $\mathrm{g} / \mathrm{m}^{2}$ & $38.8 \pm 13.9 *$ & $23.8 \pm 4.9$ \\
\hline LVEF, \% & $60.3 \pm 9.9$ & $67.1 \pm 4.4$ \\
\hline $\mathrm{LV}$ mass/BSA, $\mathrm{g} / \mathrm{m}^{2}$ & $58.3 \pm 14.4$ & $59.7 \pm 18.3$ \\
\hline RV thickness, mm & $5.8 \pm 1.5^{*}$ & $2.58 \pm 0.4$ \\
\hline IVS, $\mathrm{mm}$ & $8.3 \pm 1.5$ & $7.7 \pm 1.2$ \\
\hline TAPSE, mm & $18.9 \pm 4.4 *$ & $24.9 \pm 2.9$ \\
\hline
\end{tabular}

Data are presented as mean \pm standard deviation or median (interquartile range). ${ }^{*}$-statistically significant difference between PAH and control groups, $p<0.05$. $6 \mathrm{MWD}, 6$ min walk test distance; AcT, acceleration time; BMI, body mass index; BSA, body surface area; BNP, brain natriuretic peptide; CI, cardiac index; CHDPAH, congenital heart disease related pulmonary arterial hypertension; CTDPAH, connective tissue disease related pulmonary arterial hypertension; DPG, diastolic pulmonary gradient; $\mathrm{dPAP}$, diastolic pulmonary artery pressure; EDV, end-diastolic volume; emPAP, echo mean pulmonary artery pressure; ERA, endothelin receptor antagonist ESV, end-systolic volume; HPAH, heritable pulmonary arterial hypertension; IPAH, idiopathic pulmonary arterial hypertension; IVS, interventricular septum; LV, left ventricle; LVEF, left ventricle ejection fraction; mPAP, mean pulmonary artery pressure; MRI, magnetic resonance imaging; $\mathrm{PAH}$, pulmonary arterial hypertension; PASP, pulmonary artery systolic pressure; PAWP, pulmonary artery wedge pressure; PDE5, phosphodiesterase type 5; PVR, pulmonary vascular resistance; $\mathrm{SPAP}$, systolic pulmonary artery pressure; RAA, right atrial area; RAP right atrial pressure; RHC, right heart catheterization; RV, right ventricle; RVEF, right ventricle ejection fraction; SUV, standardized uptake value; SvO2, mixed venous oxygen saturation; TAPSE, tricuspid annular plane systolic excursion; WHO, World Health Organization. 


\subsection{Echo Parameters and RV Function}

In the PAH group, RV dimensions were significantly higher than in the control group, (Table 1). The LV ejection fraction did not differ between both groups. The mean estimated mean pulmonary arterial hypertension was $47.29 \pm 15.3 \mathrm{mmHg}$ and the mean TAPSE was $19.84 \pm 4.28 \mathrm{~mm}$. Only five patients $(17 \%)$ had severe tricuspid regurgitation. The mean pulmonary vascular resistance in RHC was $9.17 \pm 5.69 \mathrm{WU}$ and the cardiac index was $2.65 \pm 0.6 \mathrm{~L} / \mathrm{min} / \mathrm{m}^{2}$. There were significant correlations between echo-derived hemodynamic parameters and RHC-derived values, e.g., echo mPAP vs. mPAP (RHC), $\mathrm{r}=0.86, p<0.001$. In addition, MRI-derived TAPSE values correlated well with echo TAPSE, $\mathrm{r}=0.92, p<0.001$.

The echo-estimated RV ventricular-arterial coupling surrogate parameter (echo TAPSE/ sPAP) was $0.35 \pm 0.20$ and $1.51 \pm 0.22$ in the control group, $p<0.01$. This ratio was comparable to the ratio obtained with a use of MRI (TAPSE) and RHC (sPAP); as shown in the Bland-Altman plot in Figure 1.

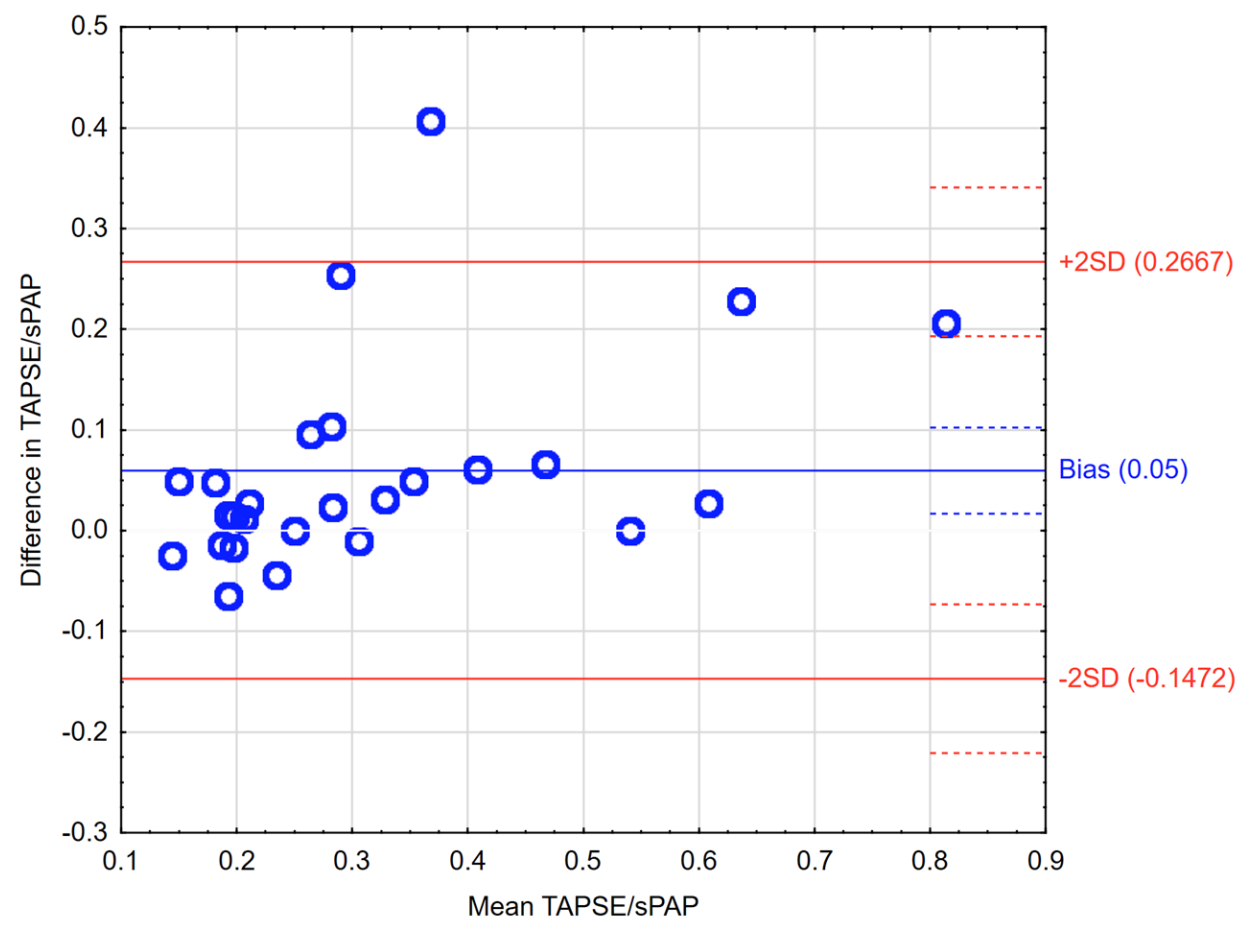

Figure 1. Bland-Altman plot presenting differences between echo-derived TAPSE/sPAP and nonecho-derived TAPSE (MRI)/sPAP (RHC) ratios.

We observed that echo TAPSE/sPAP positively correlated with RHC-derived parameters-inversely with the mPAP $(\mathrm{r}=-0.72, p<0.001)$, as shown in Figure 2A, PVR $(\mathrm{r}=-0.61, p<0.001)$ and positively with cardiac index $(\mathrm{r}=0.42, p=0.02)$. Moreover, the echo TAPSE/sPAP ratio also correlated with other MRI parameters-right ventricle ejection fraction, $\mathrm{r}=0.51, p=0.01$, as shown in Figure 2B; RV free wall thickness $(\mathrm{r}=-0.57$, $p=0.004)$; and with RV mass index $(\mathrm{r}=-0.53, p=0.01)$ in PAH. Echo TAPSE/sPAP significantly correlated with the non-echo ratio of TAPSE (MRI) /sPAP (RHC) $-r=0.75$, $p<0.005$. We did not observe significant correlations of TAPSE/sPAP ratio with other PAH prognostic factors, e.g., 6MWT distance or BNP levels. 

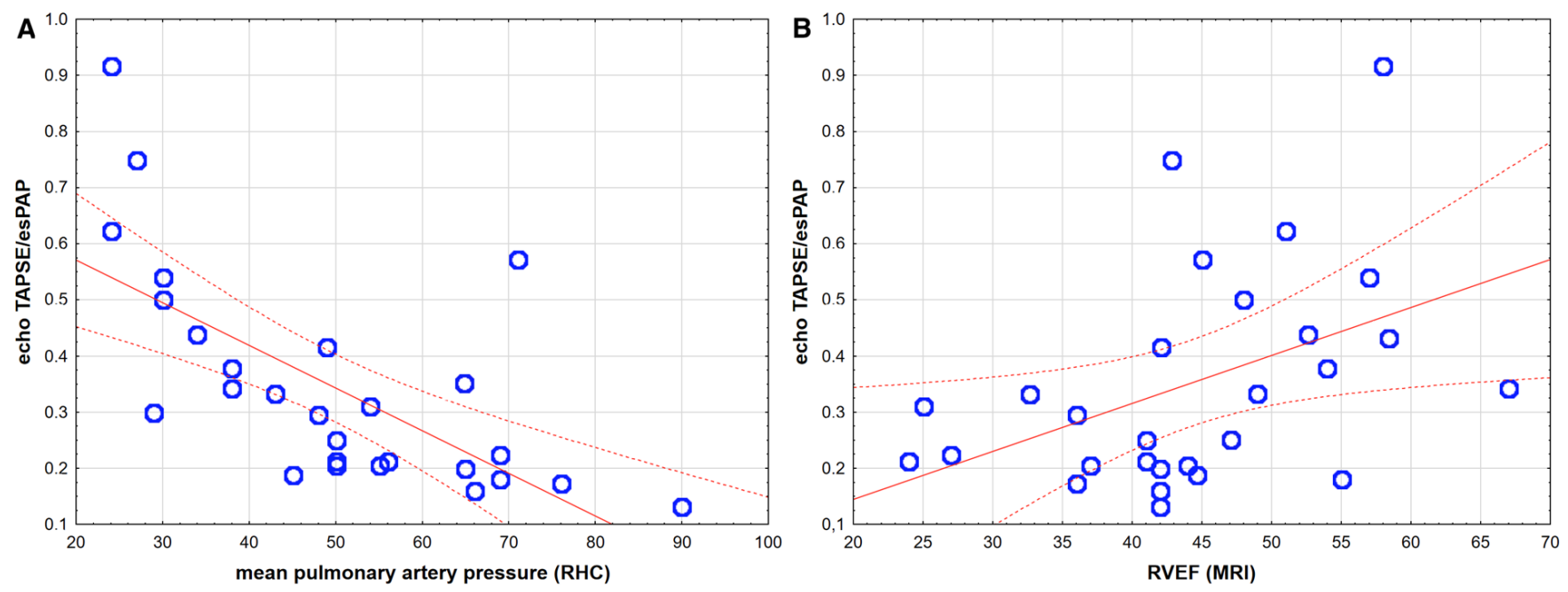

Figure 2. Spearman correlations between echo TAPSE/sPAP ratio and (A) mean pulmonary artery pressure, mPAP $(\mathrm{r}=-0.72, p<0.001)$ and $(\mathbf{B})$ right ventricle ejection fraction, $\operatorname{RVEF}(\mathrm{r}=0.51, p=0.01)$.

\subsection{Survival Analysis}

We analyzed parameters in PAH subjects who had met the combined endpointCEP $(75 \%, n=21)$. After 58 months' follow-up, six patients died, and fifteen patients had WHO class worsening with hospitalization (including three patients requiring the initiation of a parenteral prostacyclin analogue). The mean time to clinical worsening was $27.16 \pm 20.21$ months.

Patients who reached CEP had a significantly higher echo mPAP and lower TAPSE $(53.11 \pm 17.11 \mathrm{mmHg}$ vs. $34.86 \pm 8.49 \mathrm{mmHg}, p=0.006$ and $19.23 \pm 4.58 \mathrm{~mm}$ vs. $21.17 \pm 3.87 \mathrm{~mm}, p=0.06$, respectively), Table 2 .

Table 2. Comparison of PAH patients with combined endpoint (CEP) and without CEP.

\begin{tabular}{cccc}
\hline & CEP $(+)$ Patients & CEP $(-)$ Patients & p-Value \\
\hline Patients, $n$ & 21 & $62(46-258)$ & 0.60 \\
BNP, pg/mL & $269(73-410)$ & $413.6 \pm 71.4$ & 0.04 \\
6MWT distance, $\mathrm{m}$ & $394.9 \pm 92.5$ & & \\
Echo parameters & & $4.57 \pm 0.55$ & 0.50 \\
LVIDd, cm & $4.38 \pm 0.39$ & $1.24 \pm 0.82$ & 0.76 \\
IVSd, cm & $0.93 \pm 0.08$ & $0.90 \pm 0.17$ & 0.79 \\
PWd, cm & $0.91 \pm 0.13$ & $3.41 \pm 0.51$ & 0.81 \\
RVIDd, cm & $3.44 \pm 0.59$ & $20.42 \pm 6.8$ & 0.27 \\
RAA, cm ${ }^{2}$ & $24.72 \pm 8.63$ & $58.50 \pm 21.78$ & 0.12 \\
sPAP, mmHg & $72.86 \pm 32.74$ & $34.86 \pm 8.49$ & 0.03 \\
mPAP, mmHg & $53.11 \pm 17.11$ & $21.17 \pm 3.87$ & 0.06 \\
TAPSE, mm & $19.23 \pm 4.58$ & $100.71 \pm 26.49$ & 0.72 \\
AcT, ms & $98.81 \pm 32.2$ & $0.43 \pm 0.21$ & 0.04 \\
TAPSE $/$ sPAP & $0.30 \pm 0.17$ & & \\
RHC parameters & & $62.61 \pm 18.63$ & 0.04 \\
sPAP, mmHg & $85.44 \pm 28.11$ & $37.21 \pm 10.84$ & 0.006 \\
mPAP, mmHg & $54.94 \pm 16.95$ & $5.74 \pm 3.3$ & 0.01 \\
PVR, Wood Units & $10.39 \pm 5.59$ & $2.92 \pm 0.84$ & 0.01 \\
CI, L/min/m ${ }^{2}$ & $2.49 \pm 0.66$ & & \\
\hline
\end{tabular}


Table 2. Cont.

\begin{tabular}{cccc}
\hline & CEP $(+)$ Patients & CEP (-) Patients & $p$-Value \\
\hline MRI parameters & & & \\
RVEF, $\%$ & $42.05 \pm 9.53$ & $50.32 \pm 9.11$ & 0.01 \\
RV mass/BSA, g/m ${ }^{2}$ & $46.27 \pm 17.41$ & $36.71 \pm 14.25$ & 0.04 \\
RV thickness, mm & $6.22 \pm 1.35$ & $4.8 \pm 1.2$ & 0.04 \\
IVS, mm & $8.55 \pm 1.54$ & $8.77 \pm 2.43$ & 0.71 \\
TAPSE, mm & $17.72 \pm 4.14$ & $21.75 \pm 3.73$ & 0.04 \\
\hline
\end{tabular}

Data are presented as mean \pm standard deviation or median (interquartile range). $6 \mathrm{MWD}, 6$ min walk test distance; AcT, acceleration time; BMI, body mass index; BSA, body surface area; BNP, brain natriuretic peptide; CI, cardiac index; DPG, diastolic pulmonary gradient; dPAP, diastolic pulmonary artery pressure; EDV, end-diastolic volume; emPAP, echo mean pulmonary artery pressure; ESV, end-systolic volume; IVS, interventricular septum; LV, left ventricle; LVEF, left ventricle ejection fraction; mPAP, mean pulmonary artery pressure; MRI, magnetic resonance imaging; PAH, pulmonary arterial hypertension; PASP, pulmonary artery systolic pressure; PAWP, pulmonary artery wedge pressure; PVR, pulmonary vascular resistance; sPAP, systolic pulmonary artery pressure; RAA, right atrial area; RAP, right atrial pressure; RHC, right heart catheterization; RV, right ventricle; $\mathrm{RVEF}$, right ventricle ejection fraction; SUV, standardized uptake value; SvO2, mixed venous oxygen saturation; TAPSE, tricuspid annular plane systolic excursion; WHO, World Health Organization.

Interestingly, the TAPSE/sPAP ratio was significantly lower in CEP+ patients $(0.30 \pm 0.21$ vs. $0.43 \pm 0.23, p=0.04$ ).

ROC analysis confirmed the role of TAPSE/sPAP in predicting CEP (AUC 0.72 (95\% CI 0.61-0.92), $p=0.02$ ) and revealed the cut-off value $-0.25 \mathrm{~mm} / \mathrm{mmHg}$. Patients with an echo TAPSE/sPAP lower than $0.25 \mathrm{~mm} / \mathrm{mmHg}$ had worse prognosis, with log-rank test $p=0.001$, as shown in Figure 3. All six patients who died also had a TAPSE/sPAP lower than $0.25 \mathrm{~mm} / \mathrm{mmHg}$ at baseline visit.

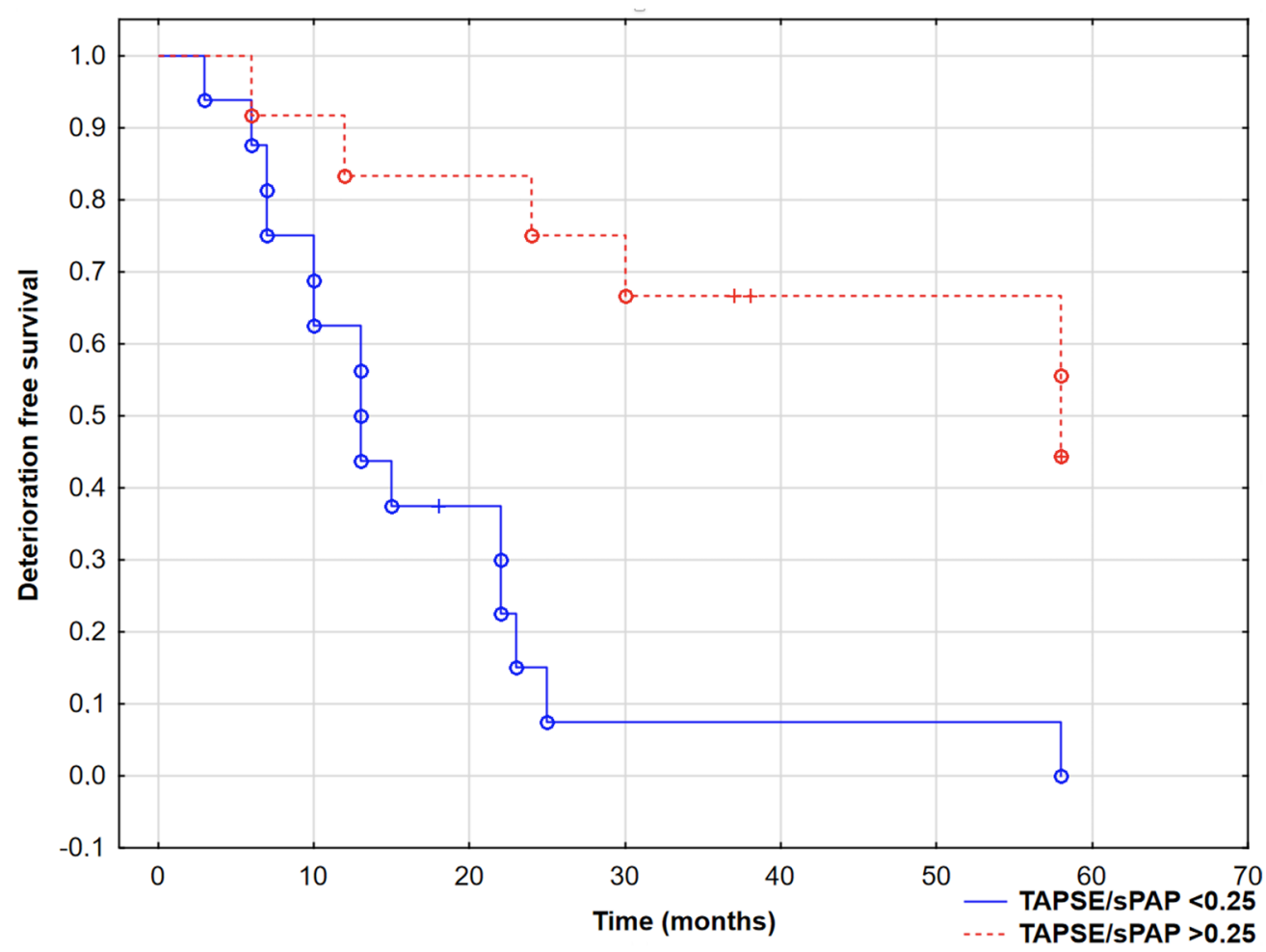

Figure 3. Kaplan-Meier curves presenting deterioration-free survival in PAH patients based on TAPSE/sPAP ratio, log-rank test, $p=0.0004 .^{\circ}{ }^{\circ}$ complete events, + - censored events.

Furthermore, ROC analysis revealed that TAPSE and sPAP alone (from echo or MRI) had a lower prediction of CEP than when presented as a ratio (according to the coupling idea), as shown in Table 3. Interestingly, the area under curve for non-echo TAPSE/sPAP 
(derived from MRI and RHC, respectively) was not significantly different from echo-only TAPSE/sPAP (0.76 [0.59-0.94] vs. 0.72 [0.61-0.92], respectively, $p=0.43)$.

Table 3. Comparison of area under curve (AUC) of various parameters for prediction of combined endpoint (ROC analysis).

\begin{tabular}{ccc}
\hline Parameter & AUC (95\% Confidence Interval) & $p$-Value \\
\hline sPAP (echo) & $0.71(0.48-0.94)$ & $p=0.06$ \\
sPAP (RHC) & $0.70(0.55-0.92)$ & $p=0.01$ \\
TAPSE (echo) & $0.65(0.39-0.92)$ & $p=0.21$ \\
TAPSE (MRI) & $0.67(0.38-0.95)$ & $p=0.23$ \\
TAPSE/sPAP (echo) & $0.72(0.61-0.92)$ & $p=0.02$ \\
TAPSE/sPAP (MRI/RHC) & $0.76(0.59-0.94)$ & $p=0.003$ \\
PVR (RHC) & $0.71(0.50-0.91)$ & $p=0.04$ \\
\hline
\end{tabular}

MRI, magnetic resonance imaging; PVR, pulmonary vascular resistance; RHC, right heart catheterization; sPAP, systemic pulmonary artery pressure; TAPSE, tricuspid annular plane systolic excursion.

\section{Discussion}

In our study, we confirmed that the simplified, echocardiographic approach of estimating RV-arterial coupling in PAH patients-the TAPSE/sPAP ratio-could be very useful in patient evaluation, even at bedside. It is linked with long-term prognosis and significantly correlates with the hemodynamic parameters of the RV from MRI and RHC.

The idea of coupling is based on the relationship between ventricular contractility and afterload. In case of elevated pulmonary vascular resistance (especially in the early stages of PAH), RV systolic function and afterload are adequately coupled; however, with a further chronic increase in afterload, the RV becomes unable to proportionally enhance its contractility, leading to progressive right heart failure [16,17].

Still, the most accurate protocol for obtaining RV-arterial coupling is direct (invasive) measurement of pressure-volume loops [3,4]. Despite their clinical value, especially in the field of $\mathrm{PAH}$, measurements of effective arterial elastance and end-systolic elastance from pressure-volume loops are not routinely obtained, due to technical difficulties. The possibility of a feasible, echocardiographic approach that mirrors relevant pathophysiological RV mechanisms was recently explored for PAH. For instance, TAPSE/mPAP or fractional area change (FAC)/ $\mathrm{mPAP}$ were found to be related to established prognostic markers of RV function [18]. According to the coupling idea, the TAPSE/sPAP ratio combines longitudinal shortening with the ability of the RV to generate pulmonary pressure. Recently, the TAPSE/sPAP ratio as a noninvasive surrogate of the RV-arterial coupling in left heart failure was confirmed by Guazzi et al. [5]. This study revealed TAPSE/sPAP as an independent predictor of event-free survival, with a value $<0.36 \mathrm{~mm} / \mathrm{mmHg}$ indicating a particularly poor prognosis, whereas in our study, this value was $<0.25 \mathrm{~mm} / \mathrm{mmHg}$ (in different study population). In another paper, Tello et al. managed to perform invasive pressure-volume loop-derived RV-arterial coupling and compare the results to echo TAPSE/sPAP [18].

In our study, we validated echo TAPSE/sPAP versus MRI- and RHC-derived parameters. As expected, the echo TAPSE/sPAP ratio strongly correlated with the hemodynamic afterload-dependent parameters, e.g., PVR or the cardiac index, which have been separately related to outcome in patients with PAH $[1,19]$. The current study presents for the first time a direct comparison of ROC curves for predicting CEP for echo TAPSE/sPAP and non-echo TAPSE/sPAP. The results presented above indicate that the TAPSE/sPAP ratio obtained non-invasively provides non-inferior prognostic information even in near-fiveyear observation.

It is noteworthy that echo, MRI and invasive measurements were performed during the baseline visit (at stable state of PAH patients), and we observed significant correlations between those parameters (Figure 2). This confirms that data used in survival analysis are reliable and represent the actual patients' state. The presented survival analysis suggests that impaired echo-estimated RV-arterial coupling may precede significant clinical 
deterioration. This technique could be routinely used during periodic monitoring visits of PAH patients.

It has to be mentioned that our study group consisted mostly of patients with idiopathic PAH in WHO III class with severely deteriorated pulmonary hemodynamics (the mean mPAP of the study group was $>40 \mathrm{mmHg}$, with the border cardiac index). The TAPSE/sPAP ratio was low overall, and therefore, we cannot directly transfer our results to patients with less severe PAH. On the other hand, patients with the lowest TAPSE/sPAP values at baseline had a worse prognosis. This could help in risk stratification by detecting the group of $\mathrm{PAH}$ patients who need therapy escalation in a shorter period of time. We are aware that sPAP is an imperfect measure of RV afterload. By definition, it combines components of both vascular resistance and pulmonary venous congestion [16], and a better option would be the pulmonary artery compliance parameter, but this requires invasive measurements. Our main goal was to validate the proposed noninvasive surrogate of $\mathrm{RV}$-arterial coupling, as it seems that the confirmation of subtle changes in RV coupling may be crucial for PAH patients' prognosis.

There are also limitations on a use of TAPSE as a measure of RV systolic function. TAPSE provides information on the longitudinal motion of the chamber (which reflects contractility), but it is highly angle- and operator-dependent [20]. In the case of severe tricuspid regurgitation, its reliability is limited; thus, TAPSE values may be overestimated. In our study, only five patients had severe tricuspid regurgitation, but it has to be mentioned that the usefulness of TAPSE may be significantly reduced in end-stage RV failure. In addition, the study group included patients with congenital heart diseases. This group of PAH patients has a better overall prognosis and survival rate, which may have had an impact on the results. Therefore, we suggest using the TAPSE/sPAP ratio merely as an additional tool during complex baseline PAH patient assessment, especially in already highrisk groups of $\mathrm{PAH}$ patients (with initially high pulmonary pressures). This additional parameter may help to detect subgroups of patients in need of rapid specific therapy escalation or qualification for lung transplantation.

TAPSE is also an afterload-dependent parameter and itself is an important predictor of poor outcome in PAH [21,22]. On the other hand, the TAPSE/sPAP ratio may be also defined as an attempt to normalize TAPSE for sPAP as an index of load adaptability; thus, this indexation theoretically creates a better parameter, specially based on the idea of coupling. Finally, when we compared ROC curves for predicting CEP, the AUC for the TAPSE/sPAP ratio is significantly higher than for TAPSE or SPAP alone.

\section{Conclusions}

The proposed echocardiographic estimation of RV ventricular-arterial coupling offers a potential easy, reliable and non-invasive prognostic parameter for the more comprehensive assessment of hemodynamic adaptation in patients with pulmonary arterial hypertension, even at bedside. It is significantly related with other invasive measurements or MRI-derived parameters. Identifying PAH patients with a nearly uncoupled right ventricle before clinical deterioration may be crucial to determining their prognosis.

Author Contributions: Conceptualization, R.K. and K.A.K.; methodology, L.A.M., R.K., M.K., B.S., and K.A.K.; data curation, R.K., E.K., P.B., K.P.-K., R.G. and K.A.K.; writing-original draft preparation, R.K. and K.A.K.; writing-review and editing, L.A.M., K.A.K.; supervision, K.A.K.; project administration, R.K. and K.A.K.; funding acquisition, R.K. and K.A.K. All authors have read and agreed to the published version of the manuscript.

Funding: This work was supported by the National Center for Science in Poland ("Preludium" grant 2017/25/N/NZ5/02689 to R.K.), statutory grant of Medical University of Bialystok and by Leading National Research Center in Bialystok.

Institutional Review Board Statement: The study was conducted according to the guidelines of the Declaration of Helsinki, and approved by the Local Bioethics Committee. Trial Registration: ClinicalTrials.gov; Protocol ID: 2017/25/N/NZ5/02689. 
Informed Consent Statement: Informed consent was obtained from all subjects involved in the study.

Conflicts of Interest: The authors declare no conflict of interest.

\section{References}

1. Galiè, N.; Hoeper, M.; Humbert, M.; Torbicki, A.; Vachiery, J.-L.; Barbera, J.A.; Beghetti, M.; Corris, P.; Gaine, S.P.; Gibbs, J.S.; et al. ESC Scientific Document Group. Guidelines for the diagnosis and treatment of pulmonary hypertension: The task force for the diagnosis and treatment of pulmonary hypertension of the European Society of Cardiology (ESC) and the European Respiratory Society (ERS), endorsed by the International Society of Heart and Lung Transplantation (ISHLT). Eur. Heart J. 2016, 37, 67-119. [PubMed]

2. Noordegraaf, A.V.; Chin, K.; Haddad, F.; Hassoun, P.M.; Hemnes, A.R.; Hopkins, S.R.; Kawut, S.M.; Langleben, D.; Lumens, J.; Naeije, R. Pathophysiology of the right ventricle and of the pulmonary circulation in pulmonary hypertension: An update. Eur. Respir. 2019, 53, 1801900. [CrossRef]

3. Noordegraaf, A.V.; Westerhof, B.E.; Westerhof, N. The relationship between the right ventricle and its load in pulmonary hypertension. J. Am. Coll. Cardiol. 2017, 69, 236-243. [CrossRef]

4. Tello, K.; Dalmer, A.; Axmann, J.; Vanderpool, R.; Ghofrani, A.; Naeije, R.; Roller, F.; Seeger, W.; Sommer, N.; Wilhelm, J.; et al. Reserve of right ventricular-arterial coupling in the setting of chronic overload. Circ. Heart Fail. 2019, 12, e005512. [CrossRef] [PubMed]

5. Guazzi, M.; Bandera, F.; Pelissero, G.; Castelvecchio, S.; Menicanti, L.; Ghio, S.; Temporelli, P.L.; Arena, R. Tricuspid annular plane systolic excursion and pulmonary arterial systolic pressure relationship in heart failure: An index of right ventricular contractile function and prognosis. Am. J. Physiol. Heart Circ. Physiol. 2013, 305, H1373-H1381. [CrossRef]

6. Guazzi, M.; Naeije, R.; Arena, R.; Corrà, U.; Ghio, S.; Forfia, P.; Rossi, A.; Cahalin, L.P.; Bandera, F.; Temporelli, P. Echocardiography of right ventriculoarterial coupling combined with cardiopulmonary exercise testing to predict outcome in heart failure. Chest 2015, 148, 226-234. [CrossRef]

7. Kazimierczyk, R.; Szumowski, P.; Nekolla, S.G.; Blaszczak, P.; Malek, L.A.; Milosz-Wieczorek, B.; Misko, J.; Jurgilewicz, D.; Hladunski, M.; Knapp, M.; et al. Prognostic role of PET/MRI hybrid imaging in patients with pulmonary arterial hypertension. Heart 2021, 107, 54-60. [CrossRef]

8. Guazzi, M. Use of TAPSE/PASP ratio in pulmonary arterial hypertension: An easy shortcut in a congested road. Int. J. Cardiol. 2018, 266, 242-244. [CrossRef]

9. Tello, K.; Axmann, J.; Ghofrani, A.; Naeije, R.; Narcin, N.; Rieth, A.; Seeger, W.; Gall, H.; Richter, M.J. Relevance of the TAPSE/PASP ratio in pulmonary arterial hypertension. Int. J. Cardiol. 2018, 266, 229-235. [CrossRef]

10. Kazimierczyk, R.; Małek, Ł.A.; Szumowski, P.; Błaszczak, P.; Jurgilewicz, D.; Hładuński, M.; Sobkowicz, B.; Myśliwiec, J.; Grzywna, R.; Musiał, W.J.; et al. Prognostic value of late gadolinium enhancement mass index in patients with pulmonary arterial hypertension. Adv. Med. Sci. 2021, 66, 28-34. [CrossRef]

11. Kazimierczyk, R.; Malek, L.A.; Szumowski, P.; Nekolla, S.G.; Blaszczak, P.; Jurgilewicz, D.; Hladunski, M.; Sobkowicz, B.; Mysliwiec, J.; Grzywna, R.; et al. Multimodal assessment of right ventricle overload-metabolic and clinical consequences in pulmonary arterial hypertension. J. Cardiovasc. Magn. Reson. 2021, 23, 49. [CrossRef]

12. Simsek, E.; Nalbantgil, S.; Ceylan, N.; Zoghi, M.; Kemal, H.S.; Engin, C.; Yagdi, T.; Ozbaran, M. Assessment of right ventricular systolic function in heart transplant patients: Correlation between echocardiography and cardiac magnetic resonance imaging. Investigation of the accuracy and reliability of echocardiography. Echocardiography 2017, 10, 1432-1438. [CrossRef] [PubMed]

13. Ptaszyńska-Kopczyńska, K.; Krentowska, A.; Sawicka, E.; Skoneczny, A.; Jasiewicz, M.; Knapp, M.; Musiał, W.J.; Sobkowicz, B.; Kamiński, K.A. The strengths and weaknesses of non-invasive parameters obtained by echocardiography and cardiopulmonary exercise testing in comparison with the hemodynamic assessment by the right heart catheterization in patients with pulmonary hypertension. Adv. Med. Sci. 2017, 1, 39-44. [CrossRef] [PubMed]

14. Aubert, R.; Venner, C.; Huttin, O.; Haine, D.; Filippetti, L.; Guillaumot, A.; Mandry, D.; Marie, P.-Y.; Juillière, Y.; Chabot, F.; et al. Three-Dimensional Echocardiography for the Assessment of Right Ventriculo-Arterial Coupling. J. Am. Soc. Echocardiogr. 2018, 8 , 905-915. [CrossRef]

15. Hsiao, S.H.; Lin, S.K.; Wang, W.C.; Yang, S.H.; Gin, P.L.; Liu, C.P. Severe tricuspid regurgitation shows significant impact in the relationship among peak systolic tricuspid annular velocity, tricuspid annular plane systolic excursion, and right ventricular ejection fraction. J. Am. Soc. Echocardiogr. 2006, 7, 902-910. [CrossRef]

16. Vonk-Noordegraaf, A.; Westerhof, N. Describing right ventricular function. Eur. Respir. J. 2013, 41, 1419-1423. [CrossRef]

17. Vanderpool, R.R.; Pinsky, M.R.; Naeije, R.; Deible, C.; Kosaraju, V.; Bunner, C.; Mathier, M.A.; Lacomis, J.; Champion, H.C.; Simon, M.A. RV-pulmonary arterial coupling predicts outcome in patients referred for pulmonary hypertension. Heart 2015, 101, 37-43. [CrossRef]

18. Tello, K.; Wan, J.; Dalmer, A.; Vanderpool, R.; Ghofrani, A.; Naeije, R.; Roller, F.; Mohajerani, E.; Seeger, W.; Herberg, U.; et al. Validation of the Tricuspid Annular Plane Systolic Excursion/Systolic Pulmonary Artery Pressure Ratio for the Assessment of Right Ventricular-Arterial Coupling in Severe Pulmonary Hypertension. Circ. Cardiovasc. Imaging 2019, 9, e009047. [CrossRef]

19. Benza, R.L.; Miller, D.P.; Gomberg-Maitland, M.; Frantz, R.P.; Foreman, A.J.; Coffey, C.S.; Frost, A.; Barst, R.J.; Badesch, D.B.; Elliott, C.G.; et al. Predicting survival in pulmonary arterial hypertension: Insights from the Registry to Evaluate Early and Long-Term Pulmonary Arterial Hypertension Disease Management (REVEAL). Circulation 2010, 2, 164-172. [CrossRef] 
20. Amsallem, M.; Sternbach, J.M.; Adigopula, S.; Kobayashi, Y.; Vu, T.A.; Zamanian, R.; Liang, D.; Dhillon, G.; Schnittger, I.; McConnell, M.V.; et al. Addressing the controversy of estimating pulmonary arterial pressure by echocardiography. J. Am. Soc. Echocardiogr. 2016, 29, 93-102. [CrossRef]

21. Kjaergaard, J.; Akkan, D.; Iversen, K.K.; Køber, L.; Torp-Pedersen, C.; Hassager, C. Right ventricular dysfunction as an independent predictor of short- and long-term mortality in patients with heart failure. Eur. J. Heart Fail. 2007, 9, 610-616. [CrossRef] [PubMed]

22. Damy, T.; Kallvikbacka-Bennett, A.; Goode, K.; Khaleva, O.; Lewinter, C.; Hobkirk, J.; Nikitin, N.P.; Dubois-Randé, J.-L.; Hittinger, L.; Clark, A.L.; et al. Prevalence of, associations with, and prognostic value of tricuspid annular plane systolic excursion (TAPSE) among out-patients referred for the evaluation of heart failure. J. Card. Fail. 2012, 18, 216-225. [CrossRef] [PubMed] 